\title{
Accelerated discovery of organic polymer photocatalysts for hydro- gen evolution from water through the integration of experiment and theory
}

\author{
Yang Bai, ${ }^{\mathrm{a}, \dagger}$ Liam Wilbraham, ${ }^{\mathrm{b}, \dagger}$ Benjamin J. Slater, ${ }^{\mathrm{a}}$ Martijn A. Zwijnenburg, ${ }^{\mathrm{b},{ }^{*}}$ Reiner Sebastian \\ Sprick, ${ }^{\mathrm{a},{ }^{*}}$ and Andrew I. Cooper ${ }^{\mathrm{a},{ }^{*}}$ \\ ${ }^{a}$ Department of Chemistry and Materials Innovation Factory, University of Liverpool, Crown Street, Liverpool, L69 \\ 7ZD, U.K. \\ ${ }^{\mathrm{b}}$ Department of Chemistry, University College London, 20 Gordon Street, London, WC1H oAJ, U.K. \\ ${ }^{\dagger}$ These two authors contributed equally.
}

Supporting Information Placeholder

\begin{abstract}
Conjugated polymers are an emerging class of photocatalysts for hydrogen production where the large breadth of potential synthetic diversity presents both an opportunity and a challenge. Here, we integrate robotic experimentation with high-throughput computation to navigate the available structure-property space. A total of 6354 copolymers was considered computationally, followed by the synthesis and photocatalytic characterization of a sub-library of more than 170 co-polymers. This led to the discovery of new polymers with sacrificial hydrogen evolution rates (HERs) of more than $6 \mathrm{mmol} \mathrm{g}^{-1} \mathrm{~h}^{-1}$. The variation in HER across the library does not correlate strongly with any single physical property but a machine learning model involving four separate properties can successfully describe up to $68 \%$ of the variation in the HER data between the different polymers. The four variables use in the model were the predicted electron affinity, the predicted ionization potential, the optical gap, and the dispersibility of the polymer particles in solution, as measured by optical transmittance.
\end{abstract}

\section{Introduction}

Hydrogen is an energy-carrier that could be a sustainable alternative to fossil fuels in the future. ${ }^{1}$ One approach for renewably generating hydrogen is direct photocatalytic water splitting, where a photocatalyst absorbs light to generate free charge-carriers. These charges, often assisted by a cocatalyst, can then reduce protons to hydrogen and either oxidize water itself (overall water splitting) or an electron donor (sacrificial water splitting). Most photocatalysts are inorganic, ${ }^{1,2}$ but in the 1990's, certain organic materials, such as oligo-/poly(p-phenylene $)^{3-5}$ and oligo-/poly(pyridine), ${ }^{6-8}$ were shown to act as hydrogen evolution photocatalysts. The subsequent demonstration of photocatalytic water splitting by carbon nitride ${ }^{9}$ in 2009 inspired a large number of studies, resulting in materials with high activities for sacrificial halfreactions, as well as reports of overall water-splitting ${ }^{10-12}$ Various sub-classes of organic photocatalysts have been studied for water splitting, including conjugated microporous polymers (CMPs), ${ }^{13-20}$ covalent triazine-based frameworks (CTFs), ${ }^{21-27}$ covalent organic frameworks (COFs), ${ }^{28-31}$ and linear conjugated polymers. ${ }^{14,32-37}$

Despite the oft-quoted advantages of synthetic tunability in organic materials, most studies involve the synthesis and characterization of a small number of chemically related polymers with limited structural diversity. Hence, only a tiny fraction of the possible chemical space for polymer photocatalysts has been explored. ${ }^{17,38}$ An alternative approach would be the high-throughput screening of many diverse copolymers. To this end, we develop a set of high-throughput techniques that integrate both experiment and computation, thus allowing the investigation of a large number of potential co-polymer photocatalysts. The development of this workflow required significant methodological development in computation, robotics, and automation to allow the testing of more than 170 materials under photocatalytic conditions.

In this first example of this approach, our experimental highthroughput workflow uses Suzuki-Miyaura polycondensation to couple a library of commercially-available dibromo arene building blocks $(\mathrm{A})$ with diboronic arene acids/acid esters (B) to prepare $\mathrm{AB}$ alternating co-polymers. The dibromo monomers underwent a limited degree of preselection to remove monomers that contained particularly reactive functional groups (e.g., acid chlorides) or monomers that might lead to cross-linked networks (e.g., iodo-bearing compounds). Apart from this, no other selection criteria or 'intuitive' selection rules were applied. The resulting dibromo compound library of 706 candidate dibromo monomers and 9 diboronic acid / acid esters (6354 candidate co-polymers in total) was screened computationally, and we chose to take forward a diverse sub-library of 127 dibromides for polymer synthesis. These were first coupled only with dibenzo[b,d]thiophene sulfone ${ }^{18,30,32,36,39,40}$ as a diboronic acid ester, giving 99 copolymers that could be isolated. Subsequently, other diboronic acid esters were investigated using a smaller set of dibromide compounds selected from the first screen giving 76 co-polymers. 

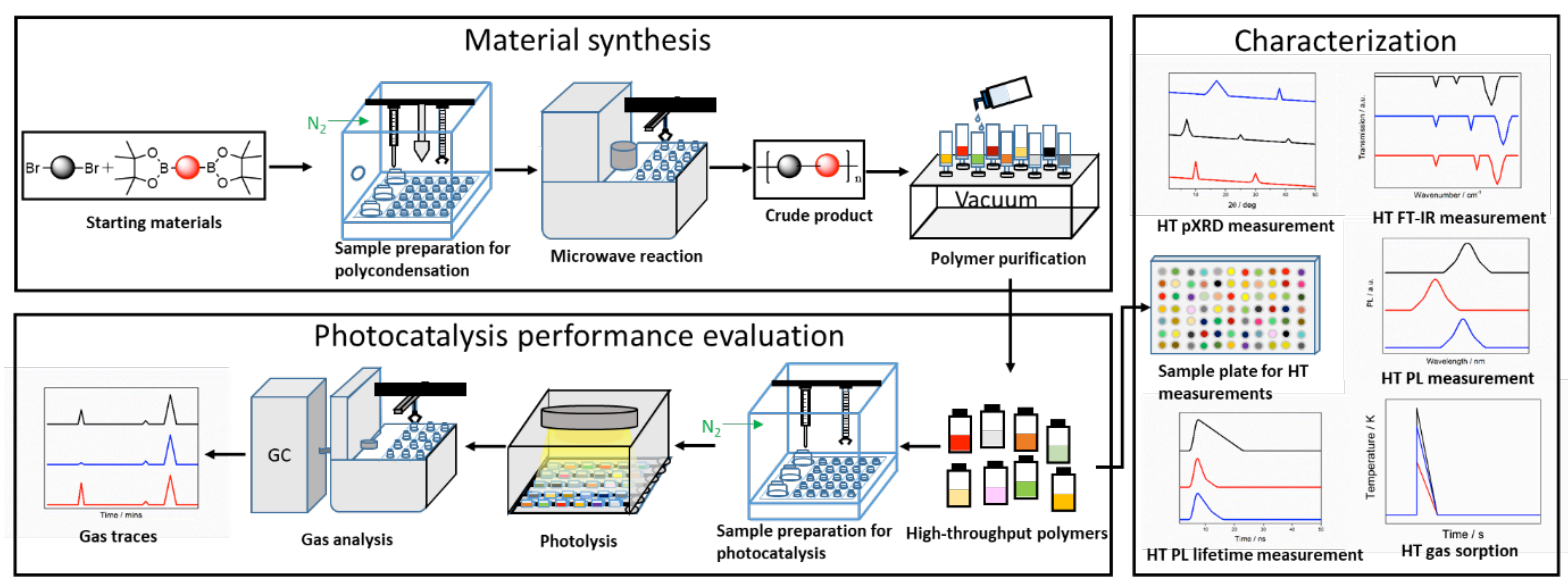

b)

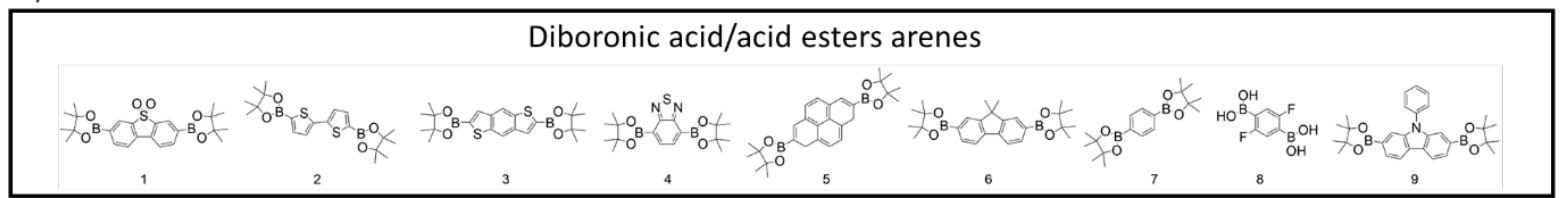

c)

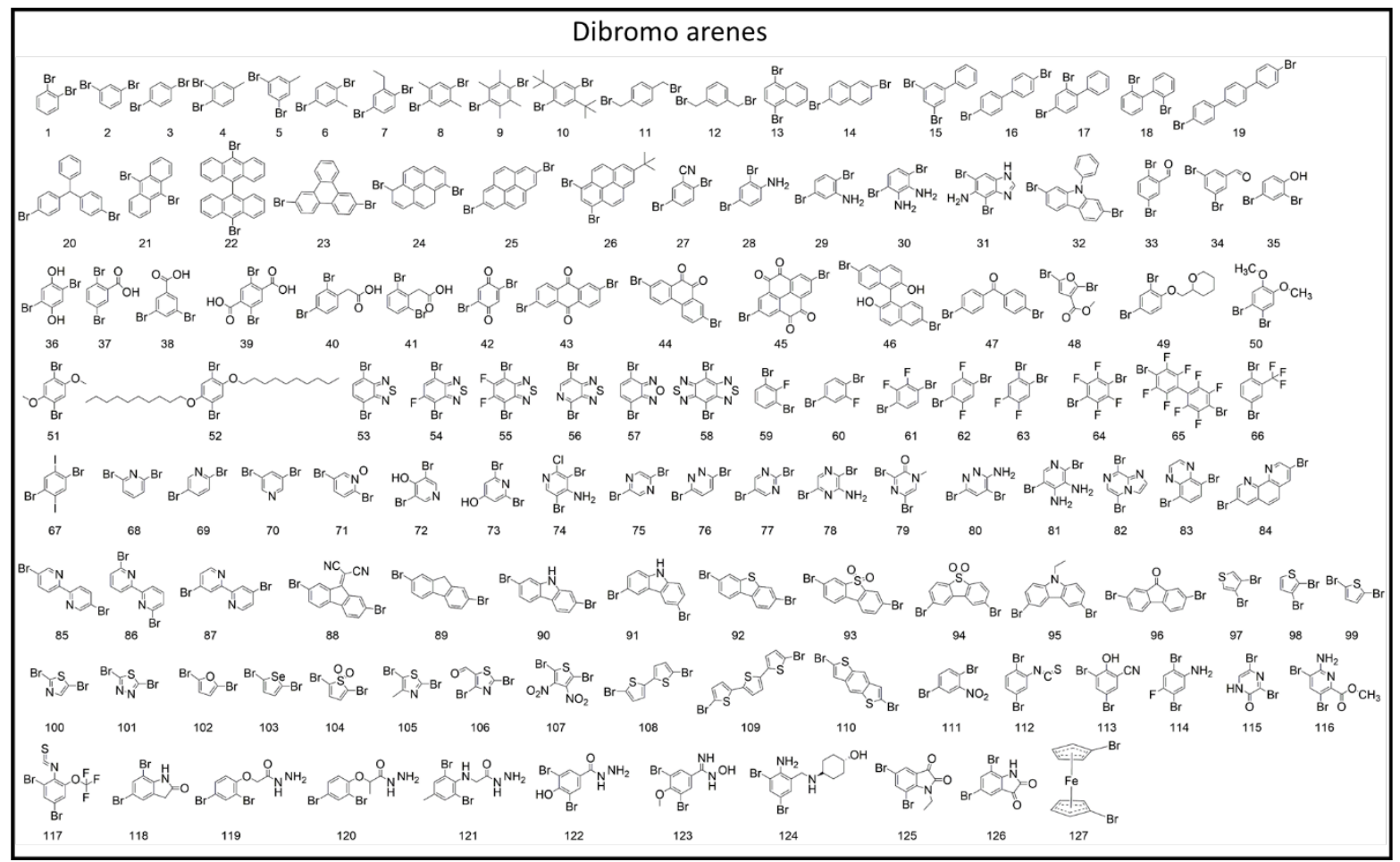

Figure 1. a) Workflow for high-throughput synthesis and property screening of the conjugated polymer library; b) diboronic acids / acid esters and c) dibromo monomers used to synthesize the co-polymers in the photocatalyst library. Note that 28 materials were not isolated or isolated in low yields and were therefore not included in the study.

By using this tiered computational-experimental strategy, the number of materials that we explored in this study significantly exceeds the total number of conjugated polymer photocatalysts described in the literature so far. Our highthroughput, data-driven approach allowed us to develop and test general hypotheses that relate co-polymer optoelectronic/material properties with catalytic activity, and to identify polymers that are among the best-performing polymer photocatalysts reported to date. We also used the large quantity of experimental data combined with machine learning techniques to test the predictability of HER based on a set of simple, measurable and/or calculable properties.

\section{Results and discussion}

To synthesize the polymer library we used microwaveassisted Suzuki-Miyaura polycondensation. ${ }^{4-43}$ 
a)

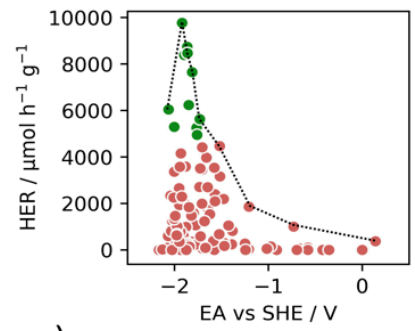

e)

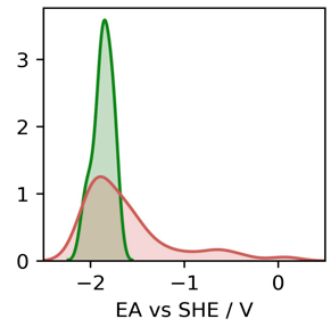

b)

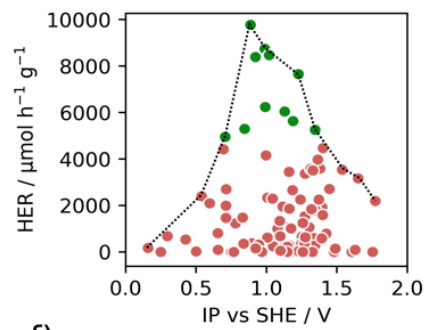

f)

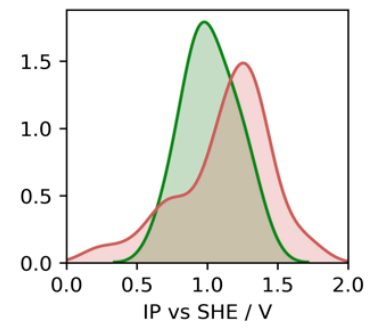

C)

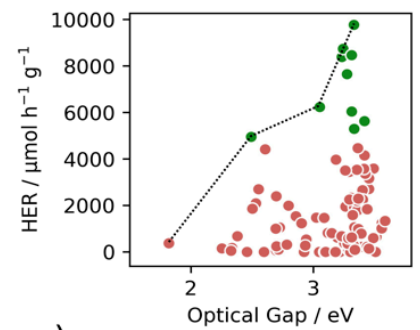

g)

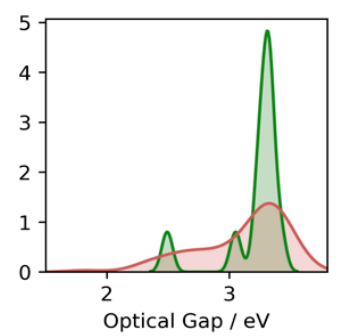

d)

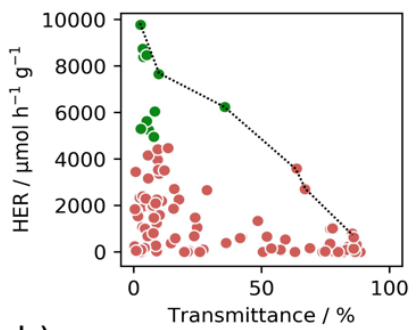

h)

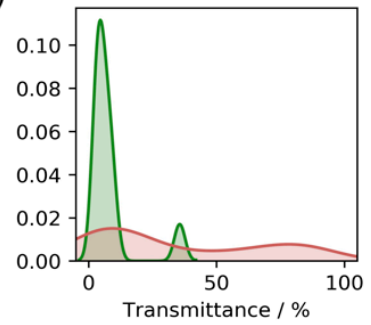

Figure 2. Photocatalytic hydrogen evolution rates (HER) of the 99-member co-polymer catalyst library in TEA/MeOH/ $\mathrm{H}_{2} \mathrm{O}$ mixture under AM ${ }_{1.5} \mathrm{G}$ illumination plotted $v s$. a) calculated EA, b) calculated IP, c) calculated optical gaps; d) experimentally measured light transmission. Kernel density estimates of distributions of e) calculated IP, f) calculated EA, g) calculated optical gap and h) light transmission. In each figure, materials with a HER greater than $50 \%$ of that of the most active polymer in the library are denoted by green points; polymers with HER less than 50\% of the most active polymer are denoted by red points. Figures a)-d) also show 'envelopes' that trace the maximum HER observed across each property rage. See Fig. S-218 for the same data when using $\mathrm{Na}_{2} \mathrm{~S}$ as electron scavenger.

A robotic formulation platform was used for weighing and loading of the microwave reactors with reagents and monomers. We first optimized the reaction conditions for poly( $p$ phenylene $)^{3,32}(\mathrm{P} 1$, see ESI). Instead of using the common catalyst $\left[\mathrm{Pd}\left(\mathrm{PPh}_{3}\right)_{4}\right]^{13,32,43,44}$ we used air-stable $\left[(\mathrm{dppf}) \mathrm{PdCl}_{2}\right]$ with tetrabutylammonium acetate acting as the base. Toluene was used instead of $N, N$-dimethylformamide (DMF) as the solvent because DMF heated too fast under microwave irradiation and this led to an excessive pressure increase. ${ }^{45}$ All materials in the library were prepared using the same conditions and without further optimization of the reaction conditions to suit a specific monomer combination.

Computational screening involved calculation of the potentials associated with the free-charge carriers in the $\mathrm{AB}$ copolymer and its optical properties by using the semiempirical density functional tight-binding xTB family of methods. ${ }^{46,47}$ We showed previously that this method gives, after calibration, results that are comparable with Density Functional Theory (DFT) at a fraction of the computational cost. ${ }^{48}$ DFT itself was found to yield accurate potentials for polymeric solids when compared to experimental ultraviolet photoelectron spectroscopy data. ${ }^{49}$ Structures for the xTB calculations were generated using the stk Python library ${ }^{50-52}$ from the curated monomer database.

All polymers were characterized by high-throughput powder X-ray diffraction (PXRD), Fourier-transform infrared spectroscopy (FT-IR), fluorescence spectroscopy (PL), and timeresolved single photon counting (TRSPC). The ability of the materials to absorb gas was also studied using a highthroughput approach, whereby the samples were exposed to $\mathrm{CO}_{2}$ under reduced pressure and the temperature change was studied using an infra-red camera. These measurements showed that, as expected, these linear polymers are essentially non-porous. We measured the photocatalytic activity for the library by using a high-throughput photoreactor system that allows the simultaneous illumination of up to 48 samples at once, with constant sample mixing. This reactor was illuminated by a solar simulator (AM1.5G, Class AAA, IEC/JIS/ASTM, 1440 W xenon, $12 \times 12$ in., MODEL: 94123A), and the gaseous products from each polymer were measured using gas chromatography (see ESI).

Initially, we focused on dibenzo[b,d]thiophene sulfone copolymers, since these materials have been shown to have high photocatalytic activities. ${ }^{18,32,36}$ We selected 127 dibenzo[b,d]thiophene sulfone co-polymers, based on the dibromo arenes shown in Fig. 1c, for synthesis from the 705 that were screened computationally and isolated 99 co-polymers, so that the range of properties predicted by computation was properly sampled. Only a small fraction $(4 \%)$ of these polymer photocatalysts have been reported previously (coupling products of monomers $2,{ }^{40} 3{ }^{32} 25^{36} 89,{ }^{36,40} 93^{39}$ with diben$\mathrm{zo}[b, d]$ thiophene sulfone); these were included as a benchmark for the new polymers. 
a)

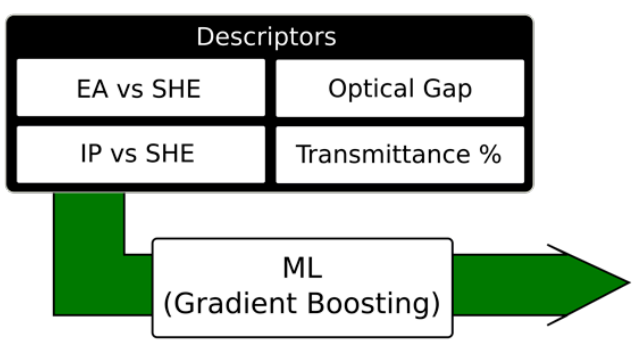

b)

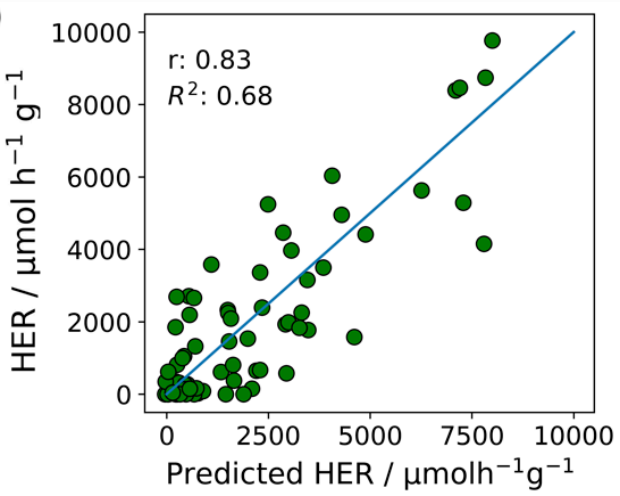

Figure 3. a) Properties used to train gradient boosting model, where IP, EA and optical gap are calculated and transmittance is measured experimentally; b) experimentally-observed HER vs. HER predicted using a gradient-boosted trees machine learning model. The model is evaluated by leave-one-out cross validation, meaning the data shown are for co-polymers not considered during

training.

Powder X-ray diffraction showed that all polymers were either amorphous or semi-crystalline. ${ }^{32} \mathrm{UV}$-visible reflectance spectra measured in the solid state showed a broad distribution of optical absorption on-sets, ranging from 400 to $650 \mathrm{~nm}$; these experimental on-sets correlate well with those predicted computationally (see Figure S-86, Figure S-125, and Figure S-159). Most of the co-polymers were fluorescent and the life-time of the excited state was estimated using timecorrelated single photon counting (TRSPC). The weighted averaged life-times ranged from $0.22 \mathrm{~ns}$ to $11.7 \mathrm{~ns}$.

The performance of the polymers as photocatalysts for hydrogen production from water was tested using the new high-throughput photoreactor, outlined above. In the absence of a scavenger, negligible hydrogen and no oxygen production was observed for all of the polymers in the library. In the presence of triethylamine (TEA) as a holescavenger, significant amounts of hydrogen were produced for many but not all of the polymers. $\mathrm{Na}_{2} \mathrm{~S}$ also acted as holescavenger for a significant number of the co-polymers in the library, but typically with lower hydrogen evolution rates compared to experiments with TEA, as observed previously. ${ }^{32}$ The reproducibility of this high-throughput approach was tested with a random selection of polymers from the library, whereby seven polymers were prepared again and their hydrogen evolution rates (HERs) were re-tested. The HERs for these repeat measurements showed a batch-to-batch variation for photocatalysis experiments in water/MeOH/TEA for the different samples of less than $15 \%$ of the overall variance in HER between the different samples in the polymer library. (See Table S-4) We also tested all polymers for photocatalytic oxygen evolution using $\mathrm{AgNO}_{3}$ as an electron scavenger using the same high-throughput photoreactor, but none of the polymers produced significant amounts of oxygen under these conditions.

Initially, all experiments were performed without any additional co-catalyst. When platinum was photo-deposited onto the materials, we observed that the rates were often enhanced (65 materials), with less active polymers (HER <1000 $\mu \mathrm{mol} \mathrm{g} \mathrm{g}^{-1}$ ) showing the largest rate enhancements of up to 26.3 times the rate without platinum. Most other materials showed an increase in HER of $10-100 \%$ in the presence of platinum. Ten materials in the library showed no change after platinum deposition (see Figure S-70), in line with some of our previous observations, ${ }^{13}$ and 17 materials showed a reduced activity (by $10-60 \%$. It is likely that entrained palladium in the material stemming from the polymerization reaction acts as a hydrogen evolution co-catalyst for the materials in the absence of platinum deposition, ${ }^{17,23,35}$ and we found between 0.07 and $1.1 \mathrm{wt}$. \% palladium via ICP-OES in the studied samples. Literature shows that the threshold concentration for palladium to act as a cocatalyst is low; $;^{35,40}$ hence, all materials may have sufficient native metal content to catalyze hydrogen production. Photocatalytic hydrogen evolution rates (HER) up to $9772 \mu \mathrm{mol} \mathrm{g}^{-1} \mathrm{~h}^{-1}$ were found for the best-performing co-polymers of dibenzo[b,d]thiophene sulfone linked to $N$-phenyl-9H-carbazole ( $\left.\mathrm{P}_{1-32}\right)$. This HER is more than four times that which we observed for the dibenzo[b,d]thiophene sulfone co-phenyl polymer, $\mathrm{P}_{7},{ }^{32,39}$ when prepared and tested under the same microwave conditions $\left(1947.9 \mu \mathrm{mol} \mathrm{g}^{-1} \mathrm{~h}^{-1}\right)$. Similar trends were obtained in kinetic runs over 4 hours using a solar simulator (see Figure S-195) and for a selection of 12 materials that were made in scale-up reactions using conventional heating instead of microwave polymerization. This shows that our high-throughput measurements are a reliable predictor for photoactive materials, and that the results can be translated to polymers that are synthesized and measured by more conventional, 'lowthroughput' methods.

The dependency of the measured HERs on a range of different computed and measured co-polymer properties is shown in Fig. 2a-2d. The various trends can be analyzed in terms of envelopes-shown as dashed lines in Fig 2-that enclose the data points for the polymer library. The measured HER shows the strongest relationship with the computed electron affinity (EA, often approximated in the literature by the energy of the LUMO) (Fig. 2a), which governs the driving force for proton reduction. For positive EA values on the Standard Hydrogen Electrode (SHE) scale, the hydrogen evolution rate is effectively zero (the one case with observable HER and a positive EA is most likely an artifact due to only limited polymerization, see section 3.9 of the ESI) and there are no polymers with computed EA values more positive than $-1.5 \mathrm{~V}$ that show high experimental HERs (HER $>50 \%$ of the most active polymer). The envelope of experimental points for HER reaches a maximum at approximately $-2 \mathrm{~V}$. It should be noted that there are also many polymers with computed EA's in this range that evolve little or no $\mathrm{H}_{2}$ (Fig 2a), illustrating 
that EA is not the only variable that governs the HER (see below). In the case of the ionization potential (IP, often approximated in the literature by the energy of the HOMO), which governs the driving force for oxidation of water or-in this case, TEA - there is a broad envelope of experimental HERs, rising with increasing IP to a peak at around $1.2 \mathrm{eV}$, before dropping again for higher IP values (Fig. 2b).

The stronger correlation between the experimental HER and the predicted EA values (Fig. 2a) compared to the predicted IP values (Fig. 2b) can be understood when considering the solution reaction potentials. At the $\mathrm{pH}$ of the TEA solution $(\mathrm{pH} \sim 11.5)$, the proton reduction potential is computed to lie at $-0.7 \mathrm{eV}$. Polymers on the right-hand side of Fig. $2 \mathrm{a}$ are therefore predicted to have negligible or no driving force for proton reduction. By contrast, all polymers are predicted to have a driving for the overall oxidation of TEA to diethylamine and acetaldehyde, the solution potential of which is also predicted to be $-0.7 \mathrm{~V}$. For most polymers, even the intermediate one-hole oxidation of TEA step (+o.7 V), which can act as a thermodynamic barrier to overall oxidation, is predicted to be exergonic.

For the computed optical gap, the shape of the envelope of measured HERs is more surprising (Fig. 2c). The most active polymers have large optical gaps, rather than small optical gaps, which one might expect to provide more effective visible light absorption. Because of the good correlation between predicted optical gap values and experimentally-measured absorption onsets (see above), the plot of measured HER vs. absorption onset looks similar (see Fig. S-78 and Fig. S-120). Plotting EA/IP vs. predicted optical gap (Fig. S-79, Fig. S-8o, Fig. S-123, Fig. S-124) shows that there is a strong correlation between EA and optical gap; that is, the polymers with the most negative EA's also have the largest optical gaps. It would seem, therefore, that the HER values of the polymers under these conditions is limited less by light absorption than by the thermodynamic driving force for proton reduction. As a result, optimization of the driving forces rather than minimization of the optical gap is probably the best strategy for maximizing the HER of these polymers, at least in the case of TEA as a sacrificial donor. This also suggests that more complex multiphase systems, where the driving forces can be decoupled from the optical gap, might be worth pursuing.

In terms of experimentally measured properties, the only property that we found with significant correlation with HER, besides absorption onset, was the light transmittance for a sample of the polymer dispersed in the TEA/MeOH/water photocatalysis mixture (Fig. 2d). The light transmittance, as measured directly after dispersing the polymer using ultrasound, ranges from $100 \%$ (indicating rapid settling or 'creaming' of material to the surface) to o\% (indicating total scattering and/or absorption of the light). As such, light transmittance is a measure of how well the polymer disperses in the reaction medium. The dispersibility of a given polymer in the reaction medium may be affected by its wettability, its physical density, and its average particle size and particle size distribution. Such properties (e.g., contact angles) are less easy to measure directly in a high-throughput fashion. The measured HER in Fig. 2 d tends to increase with decreasing transmission, and all of the most active polymers have a transmission of less than $60 \%$. This is consistent with a positive correlation between photocatalytic activity and good catalyst dispersion in the reaction mixture.

The analysis above shows how highly active polymers are localized in certain regions of property space. Clearly, there are also polymers with near-'ideal' EA, IP, optical gap and/or transmittance values that evolve little or no hydrogen. This suggests that these properties may be a necessary but insufficient condition for an active polymer photocatalyst. We therefore attempted to construct a model that could (perhaps non-linearly) relate these four properties to HER. To this end, we exploited the popular gradient boosting ${ }^{53}$ machine learning approach, as implemented in xgboost. ${ }^{54} \mathrm{We}$ combined the four properties and used leave-out cross validation to train the model to predict the experimentallyobserved HERs for the 127 dibenzo $[b, d]$ thiophene sulfone copolymers. Plotting each of the validation examples, we see that a model that combines the four properties is able to capture $68 \%$ of the variation in HER, as well as ranking the polymers, at least qualitatively, in terms of their HERs (Fig 3). It is clear that a combination of these four properties shows a much stronger correlation with hydrogen evolution rate than any one property in isolation, in line with previous evidence that photocatalytic activity is a composite property. ${ }^{33}$ The low or zero HERs for certain polymers with near-ideal EA, IP, optical gap or transmittance values can therefore be explained by the fact that one or more of its other properties lie in a less ideal region. In addition to the inherent variance (or error) in experimental HERs, estimated to correspond to maximum $15 \%$ of the variation in the HER (see above), the remaining variation that is not explained might be ascribed to a number of processes that are not described by the four properties considered by our model. These factors could include variations in charge/exciton transport, and chemical catalysis by residual palladium left over from the $\left[\mathrm{Pd}\left(\mathrm{PPh}_{3}\right)_{4}\right]$ catalyst used to prepare the polymers, to name but a few. Considering the number of potentially important factors not included in the model, it is perhaps surprising that it captures $68 \%$ of the variation in HER.

Subsequently, we considered a number of additional descriptors such as the gas uptake (a proxy for surface area), degree of crystallinity, and palladium content. However, adding these additional descriptors to the model did not increase how much of the variation in the hydrogen evolution data is captured by the model (see Fig. S217). Improved photocatalytic activity has been observed for porous photocatalysts, in particular those with wettable pores ${ }^{40}$ or mesopores photocatalysts. ${ }^{55,56}$ The materials reported are non-porous and the difference in surface areas is small. The fact that crystallinity does not seem to play an important role is at first surprising, since this can be an important factor in excition separation and charge-carrier mobility. ${ }^{57}$ Crystallinity has been invoked as an important factor in the photocatalytic performance of COFs, ${ }^{30}$ and highly crystalline carbon nitrides, ${ }^{58-60}$ in particular those with shorter stacking distances between layers. ${ }^{61}$ However, this may be material dependent and there are reports of materials where other factors, such as improved light absorption, ${ }^{62}$ or surface area, ${ }^{63}$ outweigh the lack of crystallinity in the material. 


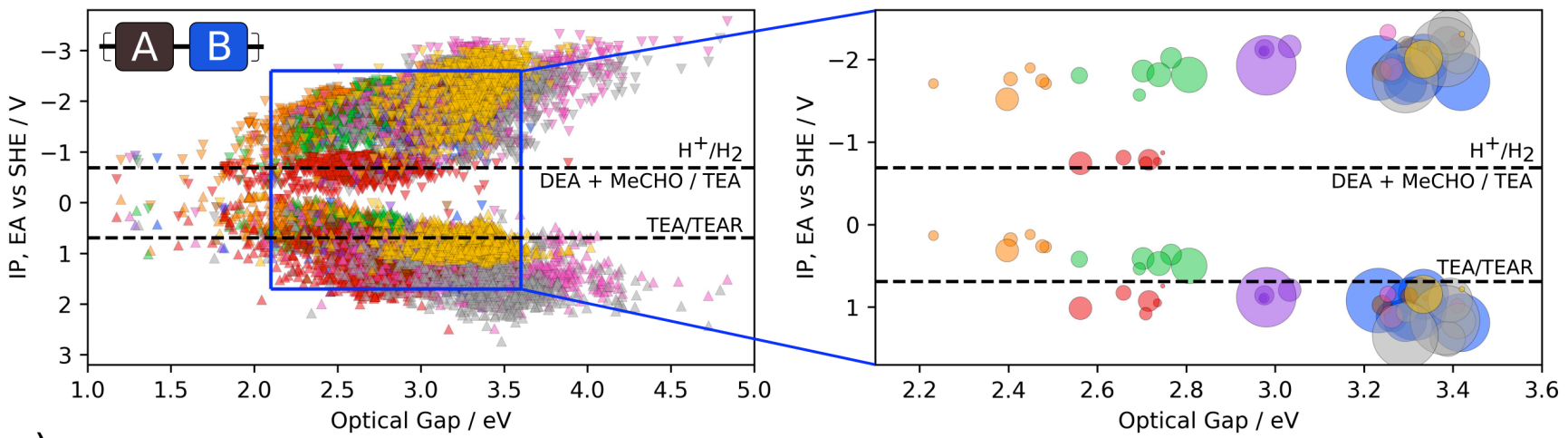

c)

\section{A Diboronic acids / acid esters}
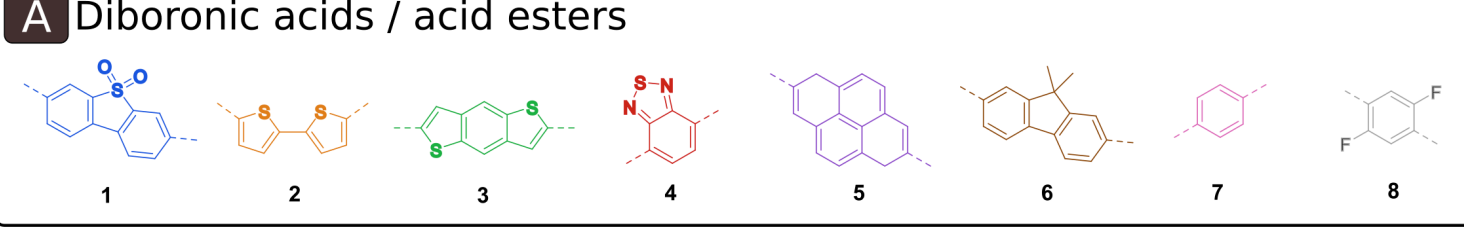

d)

e)

f)
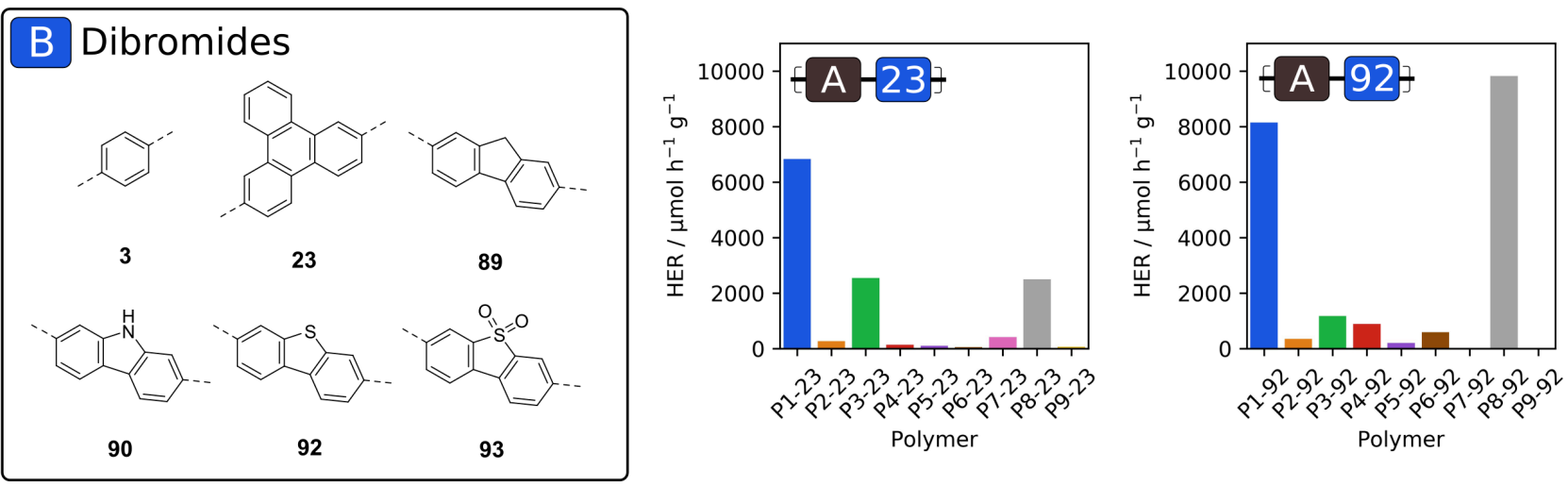

Figure 4. a) Predicted optoelectronic properties (IP, EA, optical gap) of the entire co-polymer library (6354 co-polymers) formed by the exhaustive combination of the diboronic acids/acid esters and dibromide compounds outlined in Figure ib and Figure 1c. b) Equivalent plot where marker size is proportional to the experimentally-observed HER, measured for a synthesizable subset of 43 co-polymers obtained by combining 6 dibromide compounds c) and 9 diboronic acids/acid esters d) dibromo monomers used for synthesizing the co-polymers in the photocatalyst library e) HER for triphenylene co-polymers f) HER for dibenzo[b,d]thiophene co-polymers.

Building on the above, we used computation to consider nine aryl building blocks as diboronic acid ester-monomers (eight others, plus dibenzo[b,d]thiophene sulfone, Fig $4 \mathrm{~d}$ ), coupled to all of the 706 dibromo compounds from the curated database. From this large-scale computational data $(6,354$ polymer structures in total), we see global trade-offs between optical gap, IP, and EA values, where the choice of diboronic acid ester dictates the region of property space that is accessible through co-polymerization (Fig. 4a). For example, 3,7-benzo[b,d]dithiophene copolymers (green points) and 3,3 -bithiophene copolymers (orange points) are predicted to have consistently smaller optical gaps and somewhat less positive IP values than their dibenzo[ $[b, d]$ thiophene sulfone counterparts (cyan points). By contrast, 1,4difluorophenylene co-polymers (gray points), are predicted to have, on average, larger optical gaps, more negative EA's, and more positive IP values. Benzothiadiazole co-polymers (red points) were predicted to have the least negative EA values of all of the sub-families of co-polymers considered.

To experimentally probe the influence of the aryl building block co-monomers on their photocatalytic performance, we measured the catalytic activities of polymers formed from a subset of six dibromo-monomers and these nine diboronic acid ester-monomers (Fig. 4c-d). All materials were found to be active as photocatalysts, but with HERs that ranged from 36.8 to $9828 \mu \mathrm{mol} \mathrm{g}^{-1} \mathrm{~h}^{-1}$. The variation in HER is depicted in Fig. $4 \mathrm{~b}$, where the size of circles is proportional to the measured HER values. Overall, we see similar trends for this library as we observed for the dibenzo[ $b, d]$ thiophene sulfone co-polymers discussed above. Active catalysts with higher HERs tend to have more negative EAs, more positive IPs and larger optical gaps. For example, the benzothiadiazole copolymers were all found to be among the materials with low 
activity $\left(36.8-1045.0 \mu \mathrm{mol} \mathrm{g} \mathrm{g}^{-1} \mathrm{~h}^{-1}\right)$, which is consistent with the predicted low driving forces for proton reduction in Fig. 2a. The bithiophene and benzodithophene co-polymers are also found to be somewhat less active (194.6-1049.9 $\mu \mathrm{mol} \mathrm{g}^{-1}$ $\mathrm{h}^{-1}$ ) compared to the best co-polymers, which probably is probably the result of the reduced of thermodynamic driving-force for oxidation of TEA (Fig. 4b). ${ }^{33}$ Furthermore, the analysis shown in Fig. 2 holds when polymers from this subset are included (see Figures S-154, S-156, S-157). It is likely that dispersability also plays a role: All of the bestperfoming materials display transmittance values below $\mathbf{2 0} \%$ in TEA/MeOH/water mixtures (see Figure S-117).

To study these polymers in more detail and to explore the influence of the synthesis method, we selected twelve polymers for synthesis on a larger scale using conventional heating $^{32}$ (see SI). This includes the four best-performing polymers, four polymers without any activity and four materials which lie in the middle of the activity range. Photocatalysts P64 and P62 (see Fig. 5a) were found to be the most active of these twelve polymers, with HERs of 6038.5 and $5202.6 \mu \mathrm{mol} \mathrm{g}^{-1} \mathrm{~h}^{-1}$, respectively, when scaled up and illuminated using solar simulator irradiation (AM1.5G, classification ABA, ASTME927-10). These rates are significantly higher than for previously reported $\mathrm{P}_{7}$ and $\mathrm{P}$ o under the same conditions (1171.9 and $2958.4 \mu \mathrm{mol} \mathrm{g}^{-1} \mathrm{~h}^{-1}$ ) and much higher than commercial platinized carbon nitride and platinized $\mathrm{TiO}_{2}$ from 10 vol. \% TEOA solutions (118.5 and $112.8 \mu \mathrm{mol} \mathrm{g}^{-1} \mathrm{~h}^{-1}$ ). a

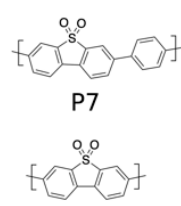

P10

C

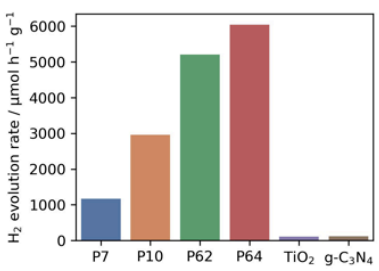

b

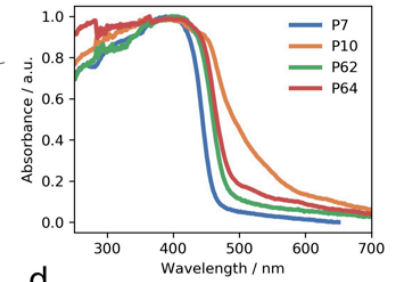

d

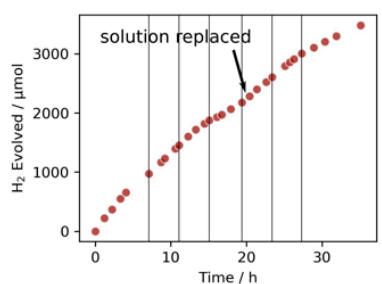

Figure 5. a) Structures of previously-reported polymer photocatalysts $\left(\mathrm{P}_{7}, \mathrm{P}_{10} \text {; top }\right)^{39}$ and the best-performing polymer photocatalysts in this study (P64, P62; bottom), as measured under identical conditions; b) UV/visible spectra of $\mathrm{P}_{7}, \mathrm{P}_{10}$, $\mathrm{P} 62$ and $\mathrm{P} 64$; c) $\mathrm{H}_{2}$ evolution rates of $\mathrm{P}_{7}, \mathrm{P}_{10}, \mathrm{P}_{2}$ and $\mathrm{P} 64$, each measurement was performed with $25 \mathrm{mg}$ catalyst in water/MeOH/triethylamine mixture under solar simulator irradiation, platinized $\mathrm{TiO}_{2}$ and platinized carbon nitride were performed with $25 \mathrm{mg}$ in water/triethanolamine (10 vol.\%) mixture under solar simulator irradiation; d) plot showing sacrificial photocatalytic hydrogen evolution versus time for P64 under solar simulator irradiation. Vertical lines indicate degassing.

The other ten materials showed activities ranging from 4621.4 to $\mathrm{o} \mu \mathrm{mol} \mathrm{g} \mathrm{g}^{-1} \mathrm{~h}^{-1}$ under these conditions. Overall, we see similar performance for the materials made via either method (S-173). The UV-visible absorption spectra were measured for these materials in the solid-state (see SI) and these were found to be comparable to the materials made on a smaller scale using microwave heating.

To evaluate the stability of these new photocatalysts, we studied the dibenzo[b,d]thiophene sulfonedibenzo[b,d]thiophene co-polymer (P64) with repeat runs under solar simulator irradiation over a total of 35 hours, with intermitted degassing and replacement of the water/MeOH/TEA mixture after 20 hours. The HER was reduced by about $10 \%$ after 10 hours, but the material was still active when irradiation was continued for a total of 35 hours (Fig. 5d). The material did not show any changes in its UV/vis, photoluminescence, or FT-IR spectrum after 35 hours of irradiation (see section S-6.12). External quantum efficiencies (EQE) of $20.7 \%$ and $\mathbf{1 5 . 1 \%}$ were measured for P64 and $\mathrm{P} 62$ at $420 \mathrm{~nm}$, which is significantly higher than previously reported EQE values for poly(p-phenylene) ( $\mathrm{P}_{1}$, $\left.\mathrm{EQE}_{420 \mathrm{~nm}}=0.4 \%\right)$, dibenzo[b,d]thiophene sulfone phenylene co-polymer $\left(\mathrm{P}_{7}, \mathrm{EQE}_{420 \mathrm{~nm}}=7.2 \%\right)$, dibenzo[b,d]thiophene sulfone homopolymer $\left(\mathrm{P}_{10}, \mathrm{EQE}_{420} \mathrm{~nm}=11.6 \%\right)^{39}$ and porous dibenzo $[b, d]$ thiophene sulfone-co-9,9'-spirobi[9H-fluorene] $\mathrm{S}_{-} \mathrm{CMP}_{3}\left(\mathrm{EQE}_{42 \mathrm{~nm}}=13.2 \%\right)^{4^{\circ}}$ under the same conditions in water/methanol/TEA mixtures, but also compared to reports of a phenyl-benzothiadiazole co-polymer $\left(\mathrm{EQE}_{420 \mathrm{~nm}}=\right.$ $4.0 \%){ }^{14}$ and 4,8-di(thiophen-2-yl)benzo[1,2- $b: 4,5^{-}$ $\left.b^{\prime}\right]$ dithiophene co-bipyridine polymer $\mathrm{PCP}_{4 \mathrm{e}}\left(\mathrm{EQE}_{350 \mathrm{~nm}}=\right.$ $1.8 \%)^{17}$

\section{Conclusions}

In summary, we have established an integrated computational and experimental high throughput approach for the screening of linear polymers as hydrogen evolution photocatalysts. We used this approach to rapidly sample chemical space and to identify high activity photocatalysts for hydrogen evolution. This generated sufficient data to explore structure-property relationships for families of closely related structures. We prepared and tested experimentally more than 170 co-polymers, most which have not been previously reported as polymer photocatalysts. Comparison of the measured HERs with computed and measured polymer properties shows that the activity of co-polymers can be rationalized in terms of the predicted electronic properties of the polymer and an experimental measurement of how well the polymer disperses in the reaction mixture. These relationships were codified in a machine learning model which explains up to $68 \%$ of the variation in the HER between the different polymers. The correlation of HER with individual properties is much weaker, supporting the notion that photocatalytic activity is a true composite property that depends on a large number of independent factors.

\section{ASSOCIATED CONTENT}

\section{Supporting Information}

Experimental details and set-ups used, spectral output of the light sources, UV-vis, FT-IR spectra, PXRD patterns, gas sorption data, hydrogen evolution data, optical transmittance data, TCSPC, SLS data, contact angle measurements, theoretical data. This material is available free of charge via the Internet at http://pubs.acs.org. 


\section{AUTHOR INFORMATION}

\section{Corresponding Authors}

aicooper@liverpool.ac.uk

ssprick@liverpool.ac.uk

m.zwijnenburg@ucl.ac.uk

\section{Notes}

The authors declare no competing financial interests.

\section{ACKNOWLEDGMENT}

We thank the Engineering and Physical Sciences Research Council (EPSRC) for financial support under Grant EP/Noo4884/1. Y.B. thanks the China Scholarship Council for a Ph.D. studentship. Dr Enrico Berardo, Dr Kim Jelfs, Dr Lukas Turcani and Dr Linjiang Chen are acknowledged for useful discussions, and Dr Christopher Kane is thanked for providing a monomer.

\section{REFERENCES}

(1) Kudo, A.; Miseki, Y. Heterogeneous Photocatalyst Materials for Water Splitting, Chem. Soc. Rev. 2009, 38, 253-278.

(2) Hisatomi, T.; Domen, K. Progress in the Demonstration and Understanding of Water Splitting using Particulate Photocatalysts, Curr. Opin. Electrochem. 2017, 2, 148-154.

(3) Yanagida, S.; Kabumoto, A.; Mizumoto, K.; Pac, C.; Yoshino, K. Poly( $p$-phenylene)-catalysed Photoreduction of Water to Hydrogen, J. Chem. Soc. Chem. Commun. 1985, o, 474-475.

(4) Shibata, T.; Kabumoto, A.; Shiragami, T.; Ishitani, O.; Pac, C.; Yanagida, S. Novel Visible-Lfght-Driven Photocatalyst. Poly(p-phenylene)-Catalyzed Photoreductions of Water, Carbonyl Compounds, and Olefins, J. Phys. Chem. 1990, 5, 2068-2076.

(5) Matsuoka, S.; Fujii, H.; Yamada, T.; Pac, C.; Ishida, A.; Takamuku, S.; Kusaba, M.; Nakashima, N.; Yanagida, S.; Hashimoto, K.; Sakata, T. Photocatalysis of Oligo(pPhenylenes): Photoreductive Production of Hydrogen and Ethanol in Aqueous Triethylamine, J. Phys. Chem. 1991, 95, 5802-5808.

(6) Yanagida, S.; Murakoshi, K.; Kusabac, M.; Nakashima, N. Synthesis of 2,2':5',2"-Terpyridine and 2,2':5',2":5",2"'Quaterpyridine and their Photocatalysis of the Reduction of Water, J. Chem. Soc., Perkin Trans. 2 1996, o, 1963-1969.

(7) Matsuoka, S.; Kohzuki, T.; Kuwana, Y.; Nakamura, A.; Yanagida, S. Visible-light-induced Photocatalysis of Poly(pyridine-2,5-diyl). Photoreduction of Water, Carbonyl Compounds and Alkenes with Triethylamine, J. Chem. Soc., Perkin Trans. 2 1992, o, 679-685.

(8) Matsuoka, S.; Kohzuki, T.; Nakamura, A.; Pac, C.; Yanagida, S. Efficient Visible-light-driven Photocatalysis. Poly(pyridine-2,5-diyl)-catalysed Hydrogen Photoevolution and Photoreduction of Carbonyl Compounds, J. Chem. Soc. Chem. Commun. 1991, 39, 580.

(9) Wang, X.; Maeda, K.; Thomas, A.; Takanabe, K.; Xin, G.; Carlsson, J. M.; Domen, K.; Antonietti, M. A Metal-Free Polymeric Photocatalyst for Hydrogen Production from Water under Visible Light, Nat. Mater. 2oo9, 8, 76-8o.

(10) Ye, S.; Wang, R.; Wu, M. Z.; Yuan, Y. P. A Review on $\mathrm{g}^{-\mathrm{C}_{3} \mathrm{~N}_{4}}$ for Photocatalytic Water Splitting and $\mathrm{CO}_{2}$ Reduction, Appl. Surf. Sci. 2015, 358, 15-27.

(11) Zhang, G.; Lan, Z. A.; Lin, L.; Lin, S.; Wang, X. Overall Water Splitting by $\mathrm{Pt} / \mathrm{g}-\mathrm{C}_{3} \mathrm{~N}_{4}$ Photocatalysts without using Sacrificial Agents, Chem. Sci. 2o16, 7, 3062-3066.

(12) Schwinghammer, K.; Mesch, M. B.; Duppel, V.; Ziegler, C.; Senker, J.; Lotsch, B. V. Crystalline Carbon Nitride Nanosheets for Improved Visible-light Hydrogen Evolution, J. Am. Chem. Soc. 2014, 136, 1730-1733.
Sprick, R. S.; Jiang, J.-X.; Bonillo, B.; Ren, S.; Ratvijitvech, T.; Guiglion, P.; Zwijnenburg, M. A.; Adams, D. J.; Cooper, A. I. Tunable Organic Photocatalysts for Visible-Light-Driven Hydrogen Evolution, J. Am. Chem. Soc. 2015, 137, 3265-3270. Yang, C.; Ma, B. C.; Zhang, L.; Lin, S.; Ghasimi, S.; Landfester, K.; Zhang, K. A. I.; Wang, X. Molecular Engineering of Conjugated Polybenzothiadiazoles for Enhanced Hydrogen Production by Photosynthesis, Angew. Chem. Int. Ed. 2016, 55, 9202-9206.

Kochergin, Y. S.; Schwarz, D.; Acharjya, A.; Ichangi, A.; Kulkarni, R.; Eliášová, P.; Vacek, J.; Schmidt, J.; Thomas, A.; Bojdys, M. J. Exploring the "Goldilocks Zone" of Semiconducting Polymer Photocatalysts by DonorAcceptor Interactions, Angew. Chem. Int. Ed. 2018, 1418814192.

(16) Li, L.; Lo, W. Y.; Cai, Z.; Zhang, N.; Yu, L. Donor-acceptor Porous Conjugated Polymers for Photocatalytic Hydrogen Production: The Importance of Acceptor Comonomer, Macromolecules 2016, 49, 6903-6909.

(17) Li, L.; Cai, Z.; Wu, Q.; Lo, W. Y.; Zhang, N.; Chen, L. X.; Yu, L. Rational Design of Porous Conjugated Polymers and Roles of Residual Palladium for Photocatalytic Hydrogen Production, J. Am. Chem. Soc. 2016, 138, 7681-7686.

(18) Wang, Z.; Yang, X.; Yang, T.; Zhao, Y.; Wang, F.; Chen, Y.; Zeng, J. H.; Yan, C.; Huang, F.; Jiang, J.-X. Dibenzothiophene Dioxide-based Conjugated Microporous Polymers for Visible-light-driven Hydrogen Production, ACS Catal. 2018, 8, 8590-8596.

(19) Schwab, M. G.; Hamburger, M.; Feng, X.; Shu, J.; Spiess, H. W.; Wang, X.; Antonietti, M.; Müllen, K. Photocatalytic Hydrogen Evolution through fully Conjugated Poly(azomethine) Networks, Chem. Commun. 2010, 46, 8932-8934.

(20) Wang, L.; Wan, Y.; Ding, Y.; Wu, S.; Zhang, Y.; Zhang, X.; Zhang, G.; Xiong, Y.; Wu, X.; Yang, J.; Xu, H. Conjugated Microporous Polymer Nanosheets for Overall Water Splitting using Visible Light, Adv. Mater. 2017, 29, 1702428.

(21) Zhang, G.; Lan, Z.-A.; Wang, X. Conjugated Polymers: Catalysts for Photocatalytic Hydrogen Evolution, Angew. Chem. Int. Ed. 2016, 55, 15712-15727.

(22) Kuecken, S.; Acharjya, A.; Zhi, L.; Schwarze, M.; Schomäcker, R.; Thomas, A. Fast Tuning of Covalent Triazine Frameworks for Photocatalytic Hydrogen Evolution, Chem. Commun. 2017, 53, 5854-5857.

(23) Meier, C. B.; Sprick, R. S.; Monti, A.; Guiglion, P.; Lee, J.-S. M.; Zwijnenburg, M. A.; Cooper, A. I. Structure-property Relationships for Covalent Triazine-based Frameworks: The Effect of Spacer Length on Photocatalytic Hydrogen Evolution from Water, Polymer 2017, 126, 283-29o.

(24) Guo, L.; Niu, Y.; Xu, H.; Li, Q.; Razzaque, S.; Huang, Q.; Tan, B.; Jin, S. Engineering Heteroatom with Atomic Precision in Donor-acceptor Covalent Triazine Frameworks to Boost Photocatalytic Hydrogen Production, J. Mater. Chem. A 2018, 40, 19775-19781.

(25) Wang, K.; Yang, L.-M.; Wang, X.; Guo, L.; Cheng, G.; Zhang, C.; Jin, S.; Tan, B.; Cooper, A. Covalent Triazine Frameworks via a Low-Temperature Polycondensation Approach. Angew. Chem. Int. Ed. 2017, 56, 14149-14153.

(26) Bi, J.; Fang, W.; Li, L.; Wang, J.; Liang, S.; He, Y.; Liu, M.; $\mathrm{Wu}$, L. Covalent Triazine-based Frameworks as Visible Light Photocatalysts for the Splitting of Water, Macromol. Rapid Commun. 2015, 36, 1799-1805.

(27) Bi, S.; Lan, Z.-A.; Paasch, S.; Zhang, W.; He, Y.; Zhang, C.; Liu, F.; Wu, D.; Zhuang, X.; Brunner, E.; Wang, X.; Zhang, F. Substantial Cyano-substituted Fully $\mathrm{sp}^{2}$-Carbon-linked Framework: Metal-Free Approach and Visible-light-driven Hydrogen Evolution, Adv. Funct. Mater. 2017, 27, 1703146.

(28) Vyas, V. S.; Haase, F.; Stegbauer, L.; Savasci, G.; Podjaski, F.; Ochsenfeld, C.; Lotsch, B. V. A Tunable Azine Covalent Organic Framework Platform for Visible Light-Induced Hydrogen Generation, Nat. Commun. 2015, 6, 8508. 
(29) Stegbauer, L.; Schwinghammer, K.; Lotsch, B. V. A Hydrazone-based Covalent Organic Framework for Photocatalytic Hydrogen Production, Chem. Sci. 2014, 5, 2789-2793.

(30) Wang, X.; Chen, L.; Chong, S. Y.; Little, M. A.; Wu, Y.; Zhu, W.-H.; Clowes, R.; Yan, Y.; Zwijnenburg, M. A.; Sprick, R. S.; Cooper, A. I. Sulfone-containing Covalent Organic Frameworks for Photocatalytic Hydrogen Evolution from Water, Nat. Chem. 2018, 10, 1180-1189.

(31) Pachfule, P.; Acharjya, A.; Roeser, J.; Langenhahn, T.; Schwarze, M.; Schomäcker, R.; Thomas, A.; Schmidt, J. Diacetylene Functionalized Covalent Organic Framework (COF) for Photocatalytic Hydrogen Generation, J. Am. Chem. Soc. 2018, 140, 1423-1427.

(32) Sprick, R. S.; Bonillo, B.; Clowes, R.; Guiglion, P.; Brownbill, N. J.; Slater, B. J.; Blanc, F.; Zwijnenburg, M. A.; Adams, D. J.; Cooper, A. I. Visible-light-driven Hydrogen Evolution Using Planarized Conjugated Polymer Photocatalysts, Angew. Chem. Int. Ed. 2016, 55, 1792-1796.

(33) Sprick, R. S.; Aitchison, C. M.; Berardo, E.; Turcani, L.; Wilbraham, L.; Alston, B. M.; Jelfs, K. E.; Zwijnenburg, M. A.; Cooper, A. I. Maximising the Hydrogen Evolution Activity in Organic Photocatalysts by Co-polymerisation, J. Mater. Chem. A 2018, 6, 11994-12003.

(34) Sprick, R. S.; Wilbraham, L.; Bai, Y.; Guiglion, P.; Monti, A.; Clowes, R.; Cooper, A. I.; Zwijnenburg, M. A. Nitrogen Containing Linear Poly(phenylene) Derivatives for Photocatalytic Hydrogen Evolution from Water, Chem. Mater. 2018, 30, 5733-5742.

(35) Kosco, J.; Sachs, M.; Godin, R.; Kirkus, M.; Francas, L.; Bidwell, M.; Qureshi, M.; Anjum, D.; Durrant, J. R.; McCulloch, I. The Effect of Residual Palladium Catalyst Contamination on the Photocatalytic Hydrogen Evolution Activity of Conjugated Polymers, Adv. Energy Mater. 2018, 8,1802181 .

(36) Dai, C.; Xu, S.; Liu, W.; Gong, X.; Panahandeh-Fard, M.; Liu, Z.; Zhang, D.; Xue, C.; Loh, K. P.; Liu, B. Dibenzothiophene$S, S$-dioxide-based Conjugated Polymers: Highly Efficient Photocatalyts for Hydrogen Production from Water under Visible Light, Small 2o18, 8, 1801839.

（37） Wang, L.; Fernández-Terán, R.; Zhang, L.; Fernandes, D. L. A.; Tian, L.; Chen, H.; Tian, H. Organic Polymer Dots as Photocatalysts for Visible Light-driven Hydrogen Generation, Angew. Chem. Int. Ed. 2016, 55, 12306-12310.

(38) Xu, Y.; Mao, N.; Zhang, C.; Wang, X.; Zeng, J.; Chen, Y.; Wang, F.; Jiang, J.-X. Rational Design of Donor- $\pi$-acceptor Conjugated Microporous Polymers for Photocatalytic Hydrogen Production, Appl. Catal. B Environ. 2018, 228, 1-9.

(39) Sachs, M.; Sprick, R. S.; Pearce, D.; Hillman, S. A. J.; Monti, A.; Guilbert, A. A. Y.; Brownbill, N. J.; Dimitrov, S.; Shi, X.; Blanc, F.; Zwijnenburg, M. A.; Nelson, J.; Durrant, J. R.; Cooper, A. I. Understanding Structure-activity Relationships in Linear Polymer Photocatalysts for Hydrogen Evolution, Nat. Commun. 2o18, 9, 4968.

(40) Sprick, R. S.; Bai, Y.; Guilbert, A. A. Y.; Zbiri, M.; Aitchison, C. M.; Wilbraham, L.; Yan, Y.; Woods, D. J.; Zwijnenburg, M. A.; Cooper, A. I. Photocatalytic Hydrogen Evolution from Water Using Fluorene and Dibenzothiophene Sulfone-Conjugated Microporous and Linear Polymers, Chem. Mater. 2019, 31, 305-313.

(41) Nehls, B. S.; Asawapirom, U.; Füldner, S.; Preis, E.; Farrell, T.; Scherf, U. Semiconducting Polymers via Microwaveassisted Suzuki and Stille Cross-coupling Reactions, $A d v$. Funct. Mater. 2004, 14, 352-356.

(42) Hoogenboom, R.; Schubert, U. S. Microwave-Assisted Polymer Synthesis: Recent Developments in a Rapidly Expanding Field of Research, Macromol. Rapid Commun. 2007, 28, 368-386.

(43) Weber, J.; Thomas, A. Toward Stable Interfaces in Conjugated Polymers: Microporous Poly(p-phenylene) and Poly(phenyleneethynylene) based on a Spirobifluorene
Building Block, J. Am. Chem. Soc. 20o8, 130, 6334-6335.

(44) Xu, Y.; Mao, N.; Feng, S.; Zhang, C.; Wang, F.; Chen, Y.; Zeng, J.; Jiang, J.-X. Perylene-containing Conjugated Microporous Polymers for Photocatalytic Hydrogen Evolution, Macromol. Chem. Phys. 2017, 218, 1-9.

(45) Ebner, C.; Bodner, T.; Stelzer, F.; Wiesbrock, F. One Decade of Microwave-Assisted Polymerizations: Quo vadis?, Macromol. Rapid Commun. 2011, 32, 254-288.

(46) Grimme, S.; Bannwarth, C. Ultra-fast Computation of Electronic Spectra for Large Systems by Tight-Binding based Simplified Tamm-Dancoff Approximation (sTDAxTB), J. Chem. Phys. 2016, 145, 054103.

(47) Grimme, S.; Bannwarth, C.; Shushkov, P. A Robust and Accurate Tight-Binding Quantum Chemical Method for Structures, Vibrational Frequencies, and Noncovalent Interactions of Large Molecular Systems Parametrized for All spd-Block Elements $(\mathrm{Z}=\mathbf{1 - 8 6})$, J. Chem. Theory Comput. 2017, 13, 1989-2009.

(48) Wilbraham, L.; Berardo, E.; Turcani, L.; Jelfs, K. E.; Zwijnenburg, M. A. High-throughput Screening Approach for the Optoelectronic Properties of Conjugated Polymers, J. Chem. Inf. Model. 2018, 58, 2450-2459.

(49) Guiglion, P.; Monti, A.; Zwijnenburg, M. A. Validating a Density Functional Theory Approach for Predicting the Redox Potentials Associated with Charge Carriers and Excitons in Polymeric Photocatalysts, J. Phys. Chem. C 2017, 121, 1498-1506.

(50) Turcani, L.; Berardo, E.; Jelfs, K. STK: A Python Toolkit for Supramolecular Assembly, J. Comput. Chem. 2018, 39, 19311942.

(51) https://github.com/JelfsMaterialsGroup/stk.

(52) Turcani, L.; Berardo, E.; Jelfs, K. E. stk: A Python Toolkit for Supramolecular Assembly, J. Comput. Chem. 2018, 39, 19311942.

(53) Chen, T.; Guestrin, C. XGBoost: A Scalable Tree Boosting System, arXiv 2016, DOI: 10.1145/2939672.2939785

(54) https://github.com/dmlc/xgboost.

(55) Wang, X.; Maeda, K.; Chen, X.; Takanabe, K.; Domen, K.; Hou, Y.; Fu, X.; Antonietti, M. Polymer Semiconductors for Artificial Photosynthesis: Hydrogen Evolution by Mesoporous Graphitic Carbon Nitride with Visible Light, J. Am. Chem. Soc. 2009, 131, 1680-1681.

(56) Chen, X.; Jun, Y.-S.; Takanabe, K.; Maeda, K.; Domen, K.; Fu, X.; Antonietti, M.; Wang, X. Ordered Mesoporous SBA15 Type Graphitic Carbon Nitride: A Semiconductor Host Structure for Photocatalytic Hydrogen Evolution with Visible Light, Chem. Mater. 2009, 21, 4093-4095.

(57) Lin, L.; Yu, Z.; Wang, X. Crystalline Carbon Nitride Semiconductors for Photocatalytic Water Splitting, Angew. Chem. Int. Ed. 2019, 58, 2-14.

(58) Guo, Y.; Li, J.; Yuan, Y.; Li, L.; Zhang, M.; Zhou, C.; Lin, Z. A Rapid Microwave-Assisted Thermolysis Route to Highly Crystalline Carbon Nitrides for Efficient Hydrogen Generation, Angew. Chem. Int. Ed. 2016, 55, 14693-14697.

(59) Bhunia, M. K.; Yamauchi, K.; Takanabe, K. Harvesting Solar Light with Crystalline Carbon Nitrides for Efficient Photocatalytic Hydrogen Evolution, Angew. Chem. Int. Ed. . 2014, 53, 11001-11005.

(6o) Li, J.; Wu, D.; Iocozzia, J.; Du, H.; Liu, X.; Yuan, Y.; Zhou, W.; Li, Z.; Xue, Z.; Lin, Z. Achieving Efficient Incorporation of $\pi$-Electrons into Graphitic Carbon Nitride for Markedly Improved Hydrogen Generation, Angew. Chem. Int. Ed. 2019, 131, 2007-2011.

(61) Zhang, G.; Li, G.; Lan, Z.-A.; Lin, L.; Savateev, A.; Heil, T.; Zafeiratos, S.; Wang, X.; Antonietti, M. Optimizing Optical Absorption, Exciton Dissociation, and Charge Transfer of a Polymeric Carbon Nitride with Ultrahigh Solar Hydrogen Production Activity, Angew. Chem. Int. Ed. 2017, 56, 1344513449.

(62) Kang, Y.; Yang, Y.; Yin, L.-C.; Kang, X.; Liu, G.; Cheng, H.M. An Amorphous Carbon Nitride Photocatalyst with 
Greatly Extended Visible-Light-Responsive Range for Photocatalytic Hydrogen Generation, Adv. Mater. 2015, 27, 4572-4577.

(63)

Schwinghammer, K.; Tuffy, B.; Mesch, M. B.; Wirnhier, E.;

Martineau, C.; Taulelle, F.; Schnick, W.; Senker, J.; Lotsch,
B. V. Triazine-based carbon nitrides for visible-light-driven hydrogen evolution, Angew. Chem. Int. Ed. 2013, 52, 24352439 .

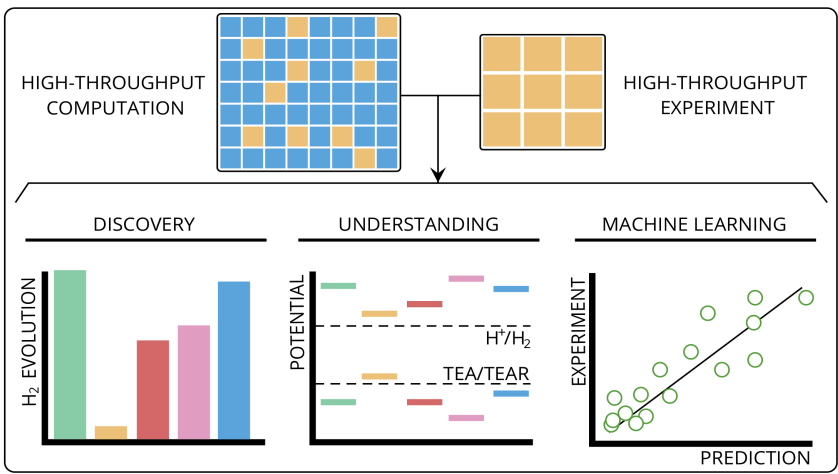

